\title{
Predictors of Disease-free Survival and Recurrence in Patients with Resected Bronchial Carcinoid Tumors
}

\author{
Paul C. Lee ${ }^{1}$ Nonso C. Osakwe ${ }^{1}$ Navnett Narula ${ }^{2}$ Jeffrey L. Port ${ }^{1}$ Subroto Paul ${ }^{1}$ Brendon M. Stiles ${ }^{1}$ \\ Weston G. Andrews ${ }^{1}$ Abu Nasar ${ }^{1}$ Nasser K. Altorki ${ }^{1}$ \\ ${ }^{1}$ Department of Cardiothoracic Surgery, Weill Cornell Medical College, \\ New York, New York, United States \\ 2 Department of Pathology, Weill Cornell Medical College, New York, \\ New York, United States \\ Address for correspondence Paul C. Lee, MD, Department of \\ Cardiothoracic Surgery, Weill Cornell Medical College, 525 East 68th \\ Street, M404 New York, New York 10065, United States \\ (e-mail: pcl9001@med.cornell.edu).
}

Thorac Cardiovasc Surg 2016;64:159-165.

\begin{abstract}
Background Bronchial carcinoids are characterized by neuroendocrine differentiation and have distinct biological behavior, recurrence patterns, and prognosis compared with adenocarcinomas or squamous cell carcinomas. Because of their often indolent nature, it has been suggested that routine postoperative imaging surveillance may not be warranted in the majority of patients. This study aims to define the factors that predict disease-free survival (DFS) and recurrence after resection of these tumors, with the goal of identifying high-risk patients for whom image surveillance may be warranted.

Methods We conducted a retrospective review of a prospective database to identify patients with completely resected bronchial carcinoid tumors. Surgical procedure, histology, pathological stage, follow-up, tumor recurrence, and survival were assessed.

Results One hundred and forty-two patients were identified. Median age was 62 years and the majority was women (106). Surgical procedures included 20 wedge resections, 10 segmentectomies, 99 lobectomies, 3 bilobectomies, 2 pneumonectomies, 6 sleeve resections, and 2 bronchectomies. Pathologic stages included I (81\%), II (10\%), III (8\%), and IV (1\%). With a median follow-up of 31 months, there were seven recurrences. The 5and 10 -year overall survival rates were $92 \%$ and $75 \%$ and DFS rates were $88 \%$ and $72 \%$, respectively. There were 34 patients with atypical carcinoids, and $6(18 \%)$ developed recurrence, compared with 1 recurrence $(1 \%)$ in the group of 108 patients with typical carcinoids $(p=0.0008)$. For atypical carcinoid tumors, the 5 - and 10 -year DFS rates were $72 \%$ and $32 \%$ versus $92 \%$ and $85 \%$ in typical carcinoid tumors $(p=0.001)$. Patients with more advanced tumor stage pT2-4 and pathologic N1/N2 nodal metastases had a significantly decreased 5- and 10-year DFS compared with those with early PT1 stage $(p=0.029)$ or those without nodal disease $(p=0.043)$. Multivariate Cox regression

Keywords

- lung cancer

- treatment

- diagnosis

- outcomes

- carcinoid analyses showed advancing age $(p=0.001)$, atypical histology $(p=0.021)$, and advanced tumor stage ( $p=0.047$ ) were significant negative predictors for DFS.

Conclusion Long-term survival after resection of bronchial carcinoids is common, especially for patients with typical carcinoid tumors. DFS can be negatively influenced by atypical histology, advanced tumor, and nodal statuses. Efforts at postoperative image surveillance should target those patients with such high-risk factors.
\end{abstract}

received

July 31,2014

accepted after revision

December 1, 2014

published online

March 10, 2015 (c) 2016 Georg Thieme Verlag KG

Stuttgart · New York
DOI http://dx.doi.org/ 10.1055/s-0035-1544211. ISSN 0171-6425. 


\section{Introduction}

Bronchial carcinoids are an uncommon group of low-grade pulmonary malignancy characterized by neuroendocrine differentiation; they make up $25 \%$ of all carcinoid tumors and $2 \%$ of all pulmonary neoplasms. ${ }^{1-3}$ Current guidelines recommend routine interval surveillance with low-dose computed tomographic (CT) scans of the chest after surgical resection for NSCLC. ${ }^{4}$ Because of the often indolent nature and uncommon development of recurrence following complete resection of bronchial carcinoid tumors, it has been suggested that routine postoperative imaging surveillance may not be warranted in the majority of those patients. ${ }^{5}$ The histological classification of bronchial carcinoid tumors into typical and atypical types identifies a subset of patients with higher tumor recurrence rate after resection. ${ }^{6-8}$ This study aims to define the factors that predict disease-free survival (DFS) and recurrence after resection of these tumors, with the goal of identifying those high-risk patients for whom CT chest surveillance should be performed.

\section{Materials and Methods}

We conducted a retrospective review of a prospective database to identify patients with primary bronchial carcinoid over a 22-year period. All patients were routinely assessed preoperatively by $\mathrm{CT}$ of the chest and upper abdomen. Positron emission tomography (PET) scan, mediastinoscopy, or Octreotide scans were performed if clinically indicated. Preoperative tissue diagnosis was obtained at the clinician's discretion through bronchoscopy and/or transthoracic needle biopsy. Hospital and office records of each patient were reviewed for demographic and clinical data, including age, sex, smoking status, and associated comorbidities. Nonsmokers were defined as patients who smoked fewer than 100 cigarettes in their lifetime. Records were also reviewed for perioperative and pathologic data, including, surgical approach, surgical procedure, 30-day operative mortality (defined as death during the same hospitalization or within 30 days after the operation), tumor size, tumor location (peripheral tumors defined as those within the outer onethird on the lung), histology, and pathologic stage. The goal of surgical resection was to achieve a complete tumor excision (R0) with negative pathologic tumor margin. When possible, lung-sparing bronchoplastic procedures were performed for central endobronchial tumors. In general, mediastinal lymph node status was assessed either by systemic lymph node dissection or sampling during surgical resection. Tumors were classified as typical or atypical carcinoids in accordance with the 2004 World Health Organization (WHO) classification guidelines. ${ }^{9}$ In patients in whom carcinoid tumors did not have such a classification in the pathology reports, the tumor slides were reviewed by a pulmonary pathologist (N. N.) and carcinoids were classified as typical or atypical. In general, history, physical examination, and low-dose surveillance CT scan of the chest and upper abdomen were done every 6 to 12 months for patients following surgical resection. Overall survival was estimated from the date of surgical resection until death from any cause. DFS was estimated from the date of surgical resection until tumor recurrence or death from any cause. Locoregional recurrence was defined as recurrence in the ipsilateral lung or mediastinal/hilar nodal stations. Distant recurrence was defined as recurrence in the contralateral lung or distant organs. Staging was done according to the TNM (tumor size-node involvement-metastasis status) classification of the seventh edition of the American Joint Commission on Cancer Staging (AJCC).

\section{Statistical Analysis}

Statistical evaluation of patients was based on demographic, surgical, and pathologic variables. Overall survival and DFS were calculated using the Kaplan-Meier method. The logrank test was used to determine the significance of group comparisons regarding these survival endpoints. Univariate and multivariate Cox regression analyses were performed to determine predictors of DFS. Univariate predictors with $p$-values less than 0.20 were selected for inclusion in the multivariate model. In the multivariate model, predictors with $p$-values equal to or less than 0.05 were deemed significant predictors. Categorical data in cross-tabulation tables were compared using Fisher's exact test or Pearson's chi-square test. Independent $t$-tests were used for two-group comparisons of continuous variables. Nonparametric data were analyzed with the Mann-Whitney U Test. SPSS version 21.0 for windows (SPSS Inc, Chicago, Illinois, United States) was used in these analyses. This study was approved by the institutional review board of the Weill Medical College of Cornell University.

\section{Results}

\section{Clinical Findings}

One hundred and forty-two patients were identified who were treated with complete surgical resection for bronchial carcinoid tumors. - Table 1 summarizes the patient demographics, surgical approach, and tumor characteristics. There were more females in this cohort and most patients were nonsmokers. The majority of patients had no symptoms on presentation. One patient had ectopic adrenocorticotropic hormone (ACTH) secretion from the carcinoid tumor and presented with Cushing syndrome that completely resolved after surgical resection. All of the patients in this cohort underwent chest radiographs, CT scans of the chest and upper abdomen to rule out liver and adrenal metastases, and flexible bronchoscopy to rule out endobronchial lesions and metastases. PET scans were done in 28 patients at clinicians' discretion and 23 had a positive finding. The low rate of PET scanning in this cohort was due to the 22-year study period and majority of the patients were presented prior to PET scan becoming a routine part of the preoperative staging workup. Octreotide scans were done at clinicians' discretion in three patients, with a positive result in two. Bone scan and brain CT or magnetic resonance imaging (MRI) were done in cases of bone pain, or neurologic symptoms or signs. Selected patients with enlarged mediastinal lymph node of greater than $1 \mathrm{~cm}$ underwent cervical mediastinoscopy to 
Table 1 Demographics, surgical approach, and tumor characteristics of 142 patients with completed resected bronchial carcinoid tumors

\begin{tabular}{|c|c|c|}
\hline Characteristics & $n$ & $\%$ \\
\hline Female & 106 & 75 \\
\hline Age (median) & $62(19-87)$ & \\
\hline Nonsmokers & 82 & 57 \\
\hline \multicolumn{3}{|l|}{ Comorbidities } \\
\hline Hypertension & 44 & 31 \\
\hline COPD & 9 & 6 \\
\hline $\mathrm{CAD} / \mathrm{MI}$ & 7 & 5 \\
\hline DM & 6 & 4 \\
\hline \multicolumn{3}{|l|}{ Surgical approach } \\
\hline VATS & 65 & 46 \\
\hline Thoracotomy & 77 & 54 \\
\hline \multicolumn{3}{|l|}{ Surgical procedure } \\
\hline Wedge resection & 20 & 14 \\
\hline Lobectomies & 99 & 70 \\
\hline Bilobectomies & 3 & 2 \\
\hline Pneumonectomies & 2 & 1.4 \\
\hline Segmentectomies & 10 & 7 \\
\hline Bronchectomies & 2 & 1.4 \\
\hline Sleeve lobectomies & 6 & 4 \\
\hline \multicolumn{3}{|l|}{ Tumor location } \\
\hline Central & 93 & 66 \\
\hline Peripheral & 49 & 34 \\
\hline \multicolumn{3}{|l|}{ Tumor histology } \\
\hline Typical & 108 & 76 \\
\hline Atypical & 34 & 24 \\
\hline \multicolumn{3}{|l|}{ Tumor pathologic stage } \\
\hline $\mathrm{IA}$ & 95 & 66.9 \\
\hline IB & 21 & 14.8 \\
\hline$I \mathrm{~A}$ & 11 & 7.7 \\
\hline IIB & 3 & 2.1 \\
\hline IIIA & 10 & 7 \\
\hline IIIB & 1 & 0.7 \\
\hline IV & 1 & 0.7 \\
\hline \multicolumn{3}{|c|}{ Tumor pathologic N-status } \\
\hline No & 120 & 84.5 \\
\hline N1 & 10 & 7 \\
\hline N2 & 10 & 7 \\
\hline $\mathrm{Nx}$ & 2 & 1.4 \\
\hline
\end{tabular}

Abbreviations: CAD/MI, coronary artery disease/myocardial infarction; COPD, chronic obstructive pulmonary disease; DM, diabetes mellitus; PET, positron emission tomography; VATS, video-assisted thoracoscopic surgery.

rule out mediastinal metastases. Presurgical tissue diagnosis was obtained as clinically indicated by transthoracic fineneedle aspiration $(n=83)$ or bronchoscopic biopsies $(n=20)$. The remaining 39 patients did not have histologic sampling prior to resection. However, most of those patients had either highly suspicious findings on CT that prompted a thoracoscopic biopsy and resection or suspicious bronchoscopic findings for endobronchial carcinoid tumors. The 
majority of resections were done by lobectomy (70\%). Bronchoplastic procedures were done in eight patients (six sleeve lobectomies and two bronchectomies). Overall, 140 patients (98.6\%) underwent careful intraoperative hilar and mediastinal lymph node dissection or sampling for accurate pathologic staging. The median length of stay in hospital after surgery was 4 days (range 2-22 days). Major postoperative complications include reintubation (1\%), pulmonary embolism (1\%), pneumonia (1\%), and renal failure requiring hemodialysis (1\%). Minor complications were prolonged air leak more than 7 days (4.4\%), urinary tract infection (1\%), and atrial arrhythmia (1.4\%). There was no 30-day operative mortality. Median length for follow-up was 31 months.

\section{Tumor Characteristics}

The tumors were classifies based on location in the lung parenchyma as central in 93 patients (66\%) and peripheral in 49 patients (34\%). Median pathologic size for resected carcinoids was $1.6 \mathrm{~cm}$. All patients had negative resection margins (R0). The majority of patients had stage I disease (82\%). Twenty patients had nodal metastases, with $\mathrm{N} 1$ disease in 10 patients and N2 disease in 10 patients. Seven of those 20 patients with nodal diseases had atypical carcinoid tumors. Overall the incidence of nodal (N1 or N2) metastases were $12.0 \%$ in patients with typical carcinoids compared with $20.6 \%$ in patients with atypical carcinoids $(p=0.258)$.

\section{Overall and Disease-Free Survival}

The overall 5- and 10-year survival rates for patients were $91.6 \%$ and $75.4 \%$, respectively ( - Fig. 1). Overall 5- and 10-year diseasefree survival (DFS) rates were $87.9 \%$ and $71.9 \%$, respectively (-Fig. 2). Patients with atypical carcinoids had a significantly decreased 5- and 10-year DFS compared with those with typical carcinoid, $72 \%$ and $32 \%$ versus $92 \%$ and $85 \%(p=0.001)$ (-Fig. 3). Patients with more advanced tumor stage pT2-4 had a significantly decreased 5- and 10-year DFS compared with those with early pT1 stage $(p=0.029)$ ( $~$ Fig. 4). Patients with pathologic N1/N2 metastases had a significantly decrease in DFS compared with those without nodal disease 5- and 10-year DFS rates were $78.4 \%$ and $39 \%$ compared with $89.6 \%$ and $79.3 \%$, respectively $(p=0.043$ ) ( $($ Fig. 5). Multivariate Cox analysis showed that advancing age, atypical histology, and advanced tumor stage pT2-4 were significant negative predictors for DFS $(p \leq 0.05)$ (-Table 2). The pN1/N2 nodal status was not a significant negative predictor for DFS by multivariate analysis.

\section{Tumor Recurrence}

A total of seven tumor recurrences developed in 142 patients after surgical resection (-Table 3 ). All tumor recurrences were confirmed by histologic sampling. Six tumor recurrences developed in patients with atypical carcinoids (6 out of 34 patients, 18\%). The rate of tumor recurrence was significantly less in those patients with typical carcinoids, with only one tumor recurrence in 108 patients (1\%) $(p=0.0008)$. Five recurrences occurred in patients with NO disease, whereas one occurred in a patient with $\mathrm{N} 1$ nodal metastasis and one occurred in patient with N2 nodal metastasis. There was no significant relationship between nodal

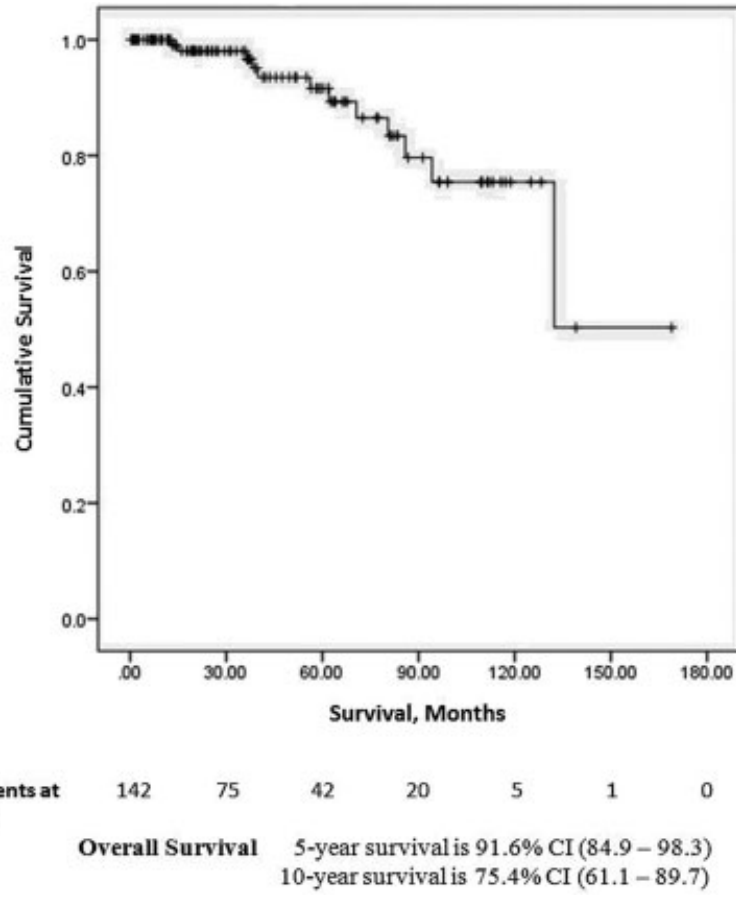

Fig. 1 Overall survival of all patients with bronchial carcinoid tumor after resection.

status and tumor recurrence $(p=0.256)$. The median tumor size of patients who developed recurrence was $3.6 \mathrm{~cm}$. Median recurrence time was 28.9 months from date of surgery. Three tumor recurrences were locoregional only, two were distant only, and two were both locoregional and distant. Treatment of locoregional recurrences mainly consisted of

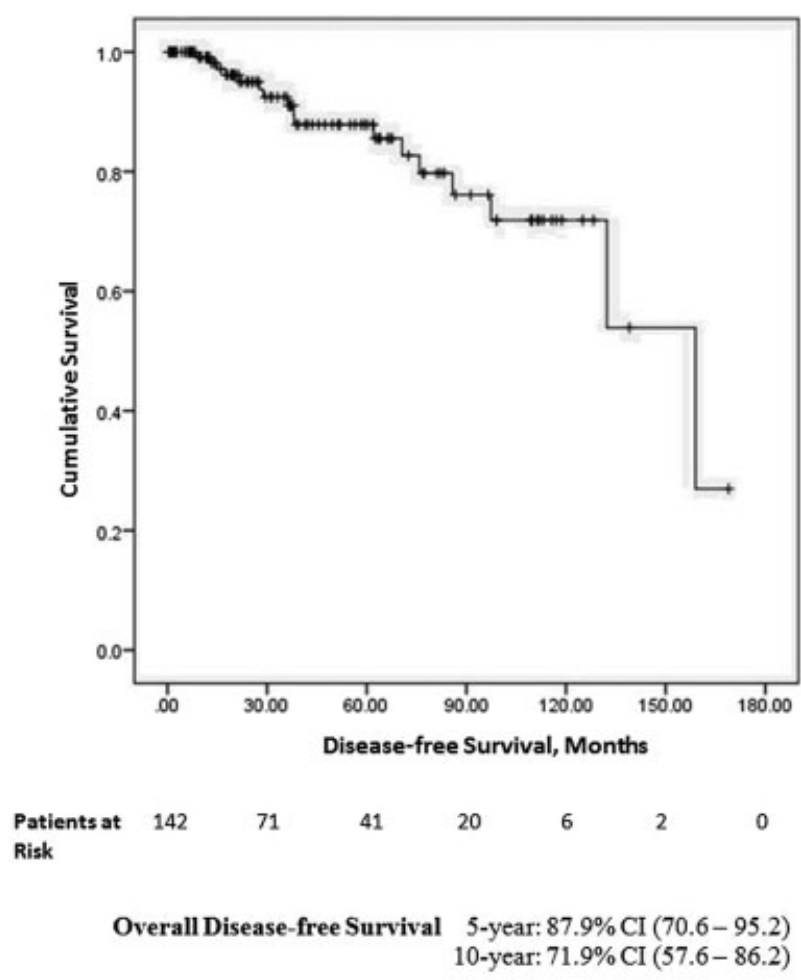

Fig. 2 Overall disease-free survival of all patients with bronchial carcinoid tumor after resection. 

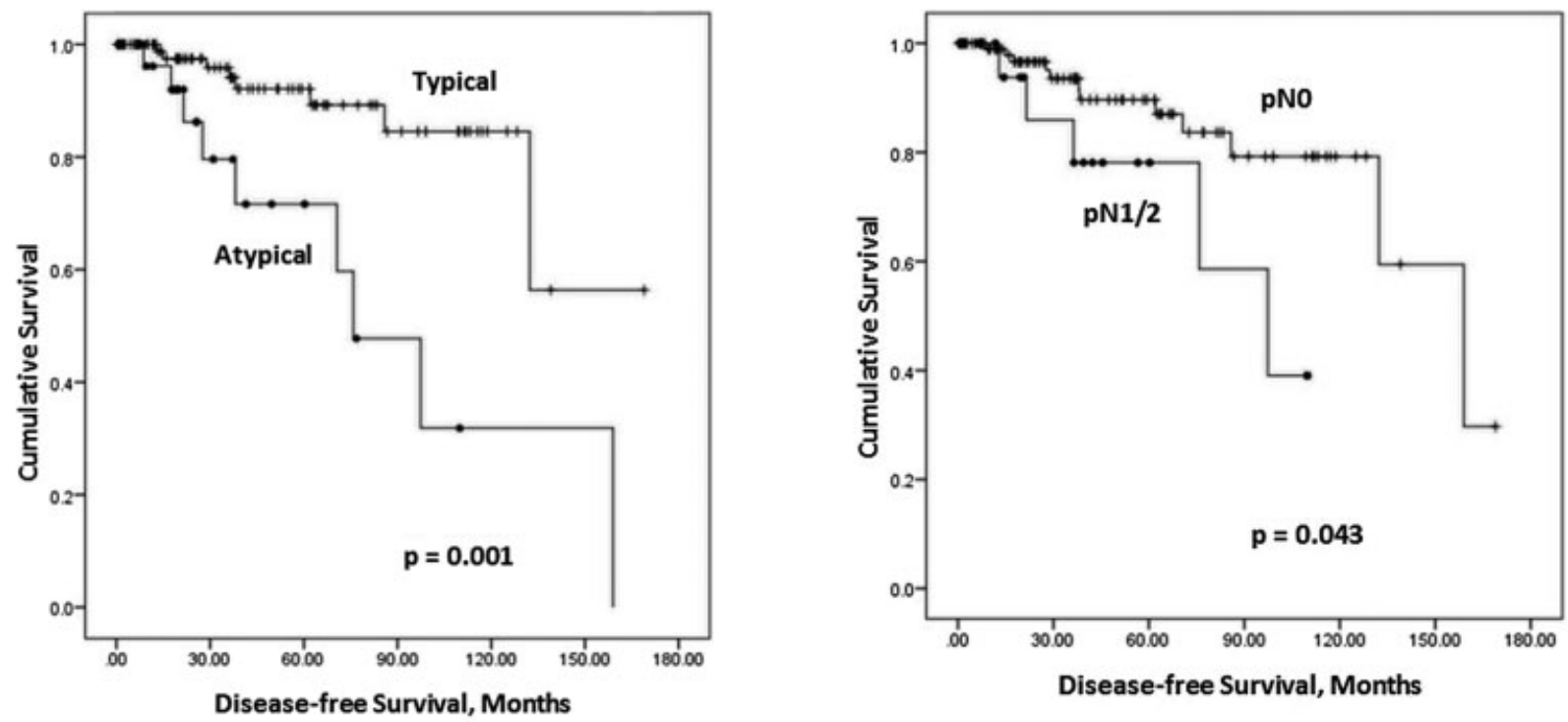

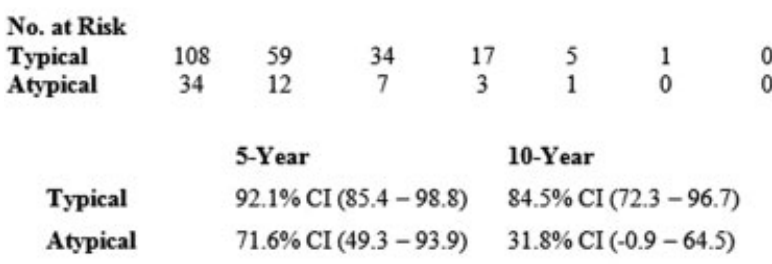

Fig. 3 Disease-free survival of patients with typical versus atypical carcinoid tumors.
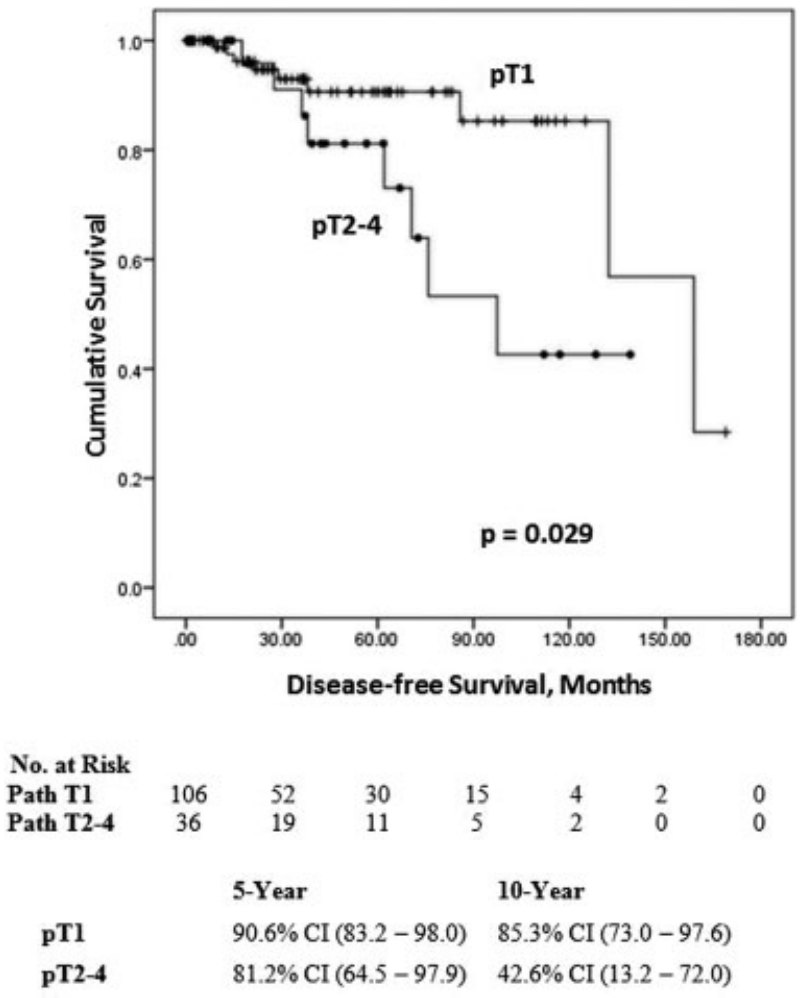

Fig. 4 Disease-free survival of patients with PT1 versus PT2-4 carcinoid tumors.

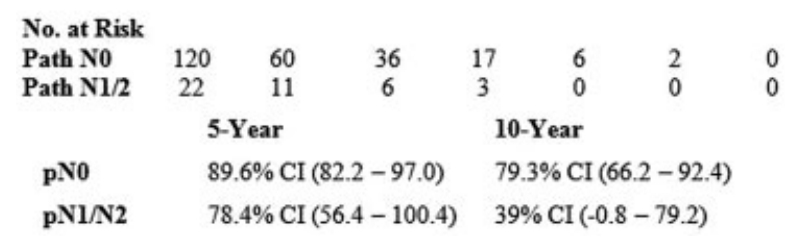

Fig. 5 Disease-free survival of patients with pNO versus $\mathrm{pN} 1 / 2$ carcinoid tumors.

radiotherapy and surgical resection, whereas treatment of those patients with distant recurrences consisted of chemotherapy or Octreotide therapy (-Table 3). One patient with liver metastases was treated with liver embolization therapy.

\section{Comment}

Bronchial carcinoids account for 2 to $5 \%$ of all primary lung malignancies and approximately $25 \%$ of all carcinoids. ${ }^{1,2,8}$ They are currently classified as neuroendocrine neoplasms by the WHO and International Association for the Study of Lung Cancer Classification. ${ }^{10}$ The rarity of this tumor is the reason why most of these case series are small and our study over a 22-year period represents one of the largest series thus far reported.

The overall survival and DFS for patients with completely resected pulmonary carcinoid tumors are good, with 5- and 10 -year overall survival of $92 \%$ and $75 \%$ and DFS of $88 \%$ and $72 \%$, respectively, similar to those reported in other studies. $^{6-8,11}$ From a prognostic standpoint, it is important to classify bronchial carcinoids into typical and atypical histological types. Our study found that atypical carcinoids had a significantly decreased 5- and 10-year survival compared with typical carcinoids, and others have shown similar findings. ${ }^{1,11-16}$ In two separate studies, the 5-year DFS for typical versus atypical carcinoid were $88.2 \%$ and $50 \%, 94.3 \%$ and $74.2 \%$, respectively. Overall, these findings support that histological classification is a significant determinant of survival. ${ }^{17,18}$ We have demonstrated in this study that significantly more tumor recurrences occurred in the atypical carcinoids compared with 
Table 2 Univariate and multivariate regression analysis for predictors of disease-free survival in patients with completely resected carcinoid tumors

\begin{tabular}{|l|l|l|l|l|}
\hline Independent variable & Univariate, HR (95\% CI) & Univariate, $p$-value & Multivariate, HR (95\% CI) & Multivariate, $p$-value \\
\hline Age & $1.091(1.033-1.154)$ & 0.002 & $1.117(1.049-1.189)$ & 0.001 \\
\hline Atypical vs. typical & $4.500(1.577-12.839)$ & 0.005 & $4.145(1.24-13.853)$ & 0.021 \\
\hline pT2-4 vs. pT1 & $3.079(1.087-8.721)$ & 0.034 & $3.544(1.014-12.383)$ & 0.047 \\
\hline pN1/N2 vs. pN0 & $2.647(0.828-8.46)$ & 0.101 & $3.107(0.766-12.598)$ & 0.112 \\
\hline
\end{tabular}

Note: Smoking status, $\mathrm{FEV}_{1}$, tumor location, procedure, and comorbidities were not significant factors in the univariate regression analysis.

Table 3 Characteristics and treatment of carcinoid tumors that recurred following complete resection

\begin{tabular}{|l|l|l|l|l|l|}
\hline TNM stage & Tumor size $(\mathbf{c m})$ & Histology & Recurrence time (mo) & $\begin{array}{l}\text { Location of } \\
\text { recurrence }\end{array}$ & Treatment \\
\hline T1aN0M0 & 0.7 & Atypical & 186.5 & Mediastinal LN & Chemo, RT \\
\hline T1bN0M0 & 2.8 & Typical & 28.9 & $\begin{array}{l}\text { Endobronchial, } \\
\text { liver }\end{array}$ & Resection, Octreotide \\
\hline T2aN0M0 & 4.0 & Atypical & 17.5 & Scapula & Chemo \\
\hline T2aN0M0 & 4.3 & Atypical & 38.1 & $\begin{array}{l}\text { Mediastinal LN, } \\
\text { spine, femur, } \\
\text { liver }\end{array}$ & Octreotide \\
\hline T2bN0M0 & 5.5 & & Hilar LN & RT \\
\hline T1aN1M0 & 1.0 & Atypical & 27.6 & Hilar LN & Chemo, RT \\
\hline T2aN2M0 & 3.6 & Atypical & 21.5 & Liver & Chemo Embolization \\
\hline
\end{tabular}

Abbreviations: LN, lymph node; RT, radiotherapy.

the typical carcinoids. Other studies have also found that atypical carcinoids are more likely to recur and are aggressive tumors biologically. $1,5,7,19$

The $\mathrm{T}$ designation of bronchial carcinoid tumors has been reported by some studies to have no statistical overall effect on survival. ${ }^{6,10}$ However, we found that carcinoid tumor with pT2-4 status have a significantly decreased 5 and 10 years DFS compared with pT1 status. Tumor status was also determined to be an important independent predictor for DFS in our multivariate analysis. Results published by others have supported our findings. ${ }^{20}$ Kaplan et al showed that stage at presentation was a significant predictor of outcome, with patients at earlier stages having better outcomes compared with those with more advanced or metastatic disease. ${ }^{21}$ It is therefore no surprise that patients with advanced tumor stages are more likely to have recurrence and spread of disease.

In this study, patients with nodal metastases had significantly worse survival. Our 5- and 10-year DFS for pN0 versus $\mathrm{pN} 1 / \mathrm{N} 2$ reported as $89 \%$ and $79 \%$ versus 78 and $39 \%$ $(p=0.043)$. Others have showed similar findings. ${ }^{8,11}$ In our current study, we classified nodal disease as present (N1/N2) or absent (N0) for statistical analysis because of the relatively small number of patients with nodal metastases $(n=20)$. This grouping is supported by published reports that showed no difference in survival between carcinoid tumor patients with $\mathrm{N} 1$ or N2 nodal disease. ${ }^{22}$ It is interesting that nodal status did not turn out to be a significant predictor for DFS on multivariate regression analyses. Even with nodal metastases, long-term survival is achievable in some patients. In our study, a patient with T1N2 had concurrent chemotherapy and radiotherapy whereas two other patients with T2N2 had chemotherapy, and these patients remained alive and disease-free for 109,39 , and 81 months, respectively. Divisi and Crisci reported treating patients with N2 disease with adjuvant chemoradiotherapy with a survival rate of $49 \%$ at 10 years. ${ }^{23}$

This study was limited by a single institutional experience and the retrospective nature of the study over two decades of study period. Because of the rarity of this tumor, a multiinstitutional study with a robust number of patients is necessary to verify our findings.

In summary, our large series showed that overall survival is good after surgical resection of bronchial carcinoid tumors. DFS can be influenced by cell type, tumor, and nodal status. Efforts on CT surveillance on patients following resection of carcinoid tumors should target those patients with high-risk features such as atypical tumor histology, advanced tumor stage, or nodal metastases.

\section{References}

1 Ferguson MK, Landreneau RJ, Hazelrigg SR, et al. Long-term outcome after resection for bronchial carcinoid tumors. Eur J Cardiothorac Surg 2000;18(2):156-161

2 Filosso PL, Rena O, Donati G, et al. Bronchial carcinoid tumors: surgical management and long-term outcome. J Thorac Cardiovasc Surg 2002;123(2):303-309 
3 Naalsund A, Rostad H, Strøm EH, Lund MB, Strand TE. Carcinoid lung tumors-incidence, treatment and outcomes: a populationbased study. Eur J Cardiothorac Surg 2011;39(4):565-569

4 Jacobson FL, Austin JH, Field JK, et al. Development of The American Association for Thoracic Surgery guidelines for low-dose computed tomography scans to screen for lung cancer in North America: recommendations of The American Association for Thoracic Surgery Task Force for Lung Cancer Screening and Surveillance. J Thorac Cardiovasc Surg 2012;144(1):25-32

5 Lou F, Sarkaria I, Pietanza C, et al. Recurrence of pulmonary carcinoid tumors after resection: implications for postoperative surveillance. Ann Thorac Surg 2013;96(4):1156-1162

6 Cao C, Yan TD, Kennedy C, Hendel N, Bannon PG, McCaughan BC. Bronchopulmonary carcinoid tumors: long-term outcomes after resection. Ann Thorac Surg 2011;91(2):339-343

7 Aydin E, Yazici U, Gulgosteren M, et al. Long-term outcomes and prognostic factors of patients with surgically treated pulmonary carcinoid: our institutional experience with 104 patients. Eur J Cardiothorac Surg 2011;39(4):549-554

8 Zhong CX, Yao F, Zhao H, Shi JX, Fan LM. Long-term outcomes of surgical treatment for pulmonary carcinoid tumors: 20 years' experience with 131 patients. Chin Med J (Engl) 2012;125(17):3022-3026

9 Travis WD, Rush W, Flieder DB, et al. Survival analysis of 200 pulmonary neuroendocrine tumors with clarification of criteria for atypical carcinoid and its separation from typical carcinoid. Am J Surg Pathol 1998;22(8):934-944

10 Cardillo G, Sera F, Di Martino M, et al. Bronchial carcinoid tumors: nodal status and long-term survival after resection. Ann Thorac Surg 2004;77(5):1781-1785

11 Jabbardarjani H, Masjedi M, Herth F. Successful treatment of endobronchial carcinoid using argon plasma coagulation. J Bronchology Interv Pulmonol 2009;16(3):196-198

12 Bertino EM, Confer PD, Colonna JE, Ross P, Otterson GA. Pulmonary neuroendocrine/carcinoid tumors: a review article. Cancer 2009; 115(19):4434-4441
13 Neyman K, Sundset A, Naalsund A, et al. Endoscopic treatment of bronchial carcinoids in comparison to surgical resection: a retrospective study. J Bronchology Interv Pulmonol 2012; 19(1):29-34

14 Johnson R, Trocha S, McLawhorn M, et al. Histology, not lymph node involvement, predicts long-term survival in bronchopulmonary carcinoids. Am Surg 2011;77(12):1669-1674

15 Bini A, Brandolini J, Cassanelli N, et al. Typical and atypical pulmonary carcinoids: our institutional experience. Interact Cardiovasc Thorac Surg 2008;7(3):415-418

16 Fiala P, Petrásková K, Cernohorský S, Kinkor Z, Krepela E, Zatloukal P. Bronchial carcinoid tumors: long-term outcome after surgery. Neoplasma 2003;50(1):60-65

17 Machuca TN, Cardoso PF, Camargo SM, et al. Surgical treatment of bronchial carcinoid tumors: a single-center experience. Lung Cancer 2010;70(2):158-162

18 Okoye CC, Jablons DM, Jahan TM, Kukreja J, Cardozo S, Yom SS. Divergent management strategies for typical versus atypical carcinoid tumors of the thoracic cavity. Am J Clin Oncol 2014;37(4): 350-355

19 Filosso P, Oliaro A, Ruffini E, et al. Outcome and prognostic factor in bronchial carcinoids. J Thorac Oncol 2013;8: $1282-1288$

20 Harpole DH Jr, Feldman JM, Buchanan S, Young WG, Wolfe WG. Bronchial carcinoid tumors: a retrospective analysis of 126 patients. Ann Thorac Surg 1992;54(1):50-54, discussion 54-55

21 Kaplan B, Stevens CW, Allen P, Liao Z, Komaki R. Outcomes and patterns of failure in bronchial carcinoid tumors. Int J Radiat Oncol Biol Phys 2003;55(1):125-131

22 Martini N, Zaman MB, Bains MS, et al. Treatment and prognosis in bronchial carcinoids involving regional lymph nodes. J Thorac Cardiovasc Surg 1994;107(1):1-6, discussion 6-7

23 Divisi D, Crisci R. Carcinoid tumors of the lung and multimodal therapy. Thorac Cardiovasc Surg 2005;53(3):168-172 